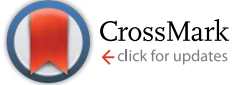

Cite this: RSC Adv., 2016, 6, 63064
Received 27th May 2016

Accepted 23rd June 2016

DOI: $10.1039 / c 6 r a 13823 c$

www.rsc.org/advances

\section{Adsorption of natural surface active compounds obtained from corn on human hair}

\author{
M. Rincón-Fontán, $\uparrow^{a}$ L. Rodríguez-López, $\uparrow^{a} X$ Xecino, $\uparrow^{a b} \mathrm{~J} . M$. Cruz $\uparrow^{a}$ \\ and A. B. Moldestª
}

In this work, an aqueous solution containing surface-active compounds, extracted from corn steep liquor (CSL), was added to human hair and its adsorption was studied by applying an incomplete factorial design. The independent variables established in the study were temperature $\left(20-50^{\circ} \mathrm{C}\right), \mathrm{pH}(5-7)$ and treatment time (2-30 $\mathrm{min})$; whereas the dependent variable studied was the adsorption capacity of hair. It was observed that the adsorption of the biosurfactant onto hair was very fast, occurring few minutes after starting the experiments. The time was, in the range studied, the least significant independent variable while temperature had an important effect on the adsorption of this biosurfactant onto hair. It was observed that the capacity of hair to adsorb the biosurfactant was improved at low temperatures. Moreover, $\mathrm{pH}$ had an intermediate significant effect, mainly at middle or high temperatures, meanwhile at low temperatures, the $\mathrm{pH}$, in the range studied, almost did not affect the adsorption process. At the higher concentration of biosurfactant, hair was able to adsorb the biosurfactant with a maximum capacity of $3679 \mu \mathrm{g} \mathrm{g}^{-1}$.

\section{Introduction}

Surfactants are important ingredients included in the formulation of hair care products. For instance, shampoos are composed of 10 to 30 ingredients including surfactants, conditioning agents, special care ingredients, and additives. ${ }^{1}$ Surfactants are usually used to modify the hair surface properties at the same time which contribute to remove contaminants or sebum from hair. ${ }^{2,3}$

Human hair is composed by proteins, lipids, water and oligoelements. The total amount of lipid extractable from hair is between 1 and $9 \%$ of the weight of the hair; whereas protein represents the $65-95 \%$ of hair composition. ${ }^{4}$ Some authors have analyzed different human hair and they found that palmitic and palmitoleic acid are the major fatty acids, followed by oleic and stearic acid. ${ }^{5}$ Although the amount of lipids is not so high, these play an important role in hair fibers: they prevent hair dryness, avoid the lower scalp hair density, growth rate and hair fiber diameters. In hair can exist two kinds of lipids: bound and free lipids. The first ones are covalently bonded to the proteins attached to the cuticle surface of hair, and they prevent the losses of proteins; ${ }^{4}$ whereas free lipids are between the keratin fibers surface and serve to decrease the isoelectric point of hair,

${ }^{a}$ Chemical Engineering Department, School of Industrial Engineering (EEI), University of Vigo, Campus As Lagoas-Marcosende, 36310 Vigo-Pontevedra, Spain. E-mail: amoldes@uvigo.es; Tel: +34986812022

${ }^{b}$ CEB-Centre of Biological Engineering, University of Minho, Campus de Gualtar, 4710057 Braga, Portugal

$\uparrow$ The authors declare no competing financial interest. improving the binding of conditioner ingredients on hair surface layers. Therefore, these free fatty acids are also important for the protection of hair. ${ }^{6}$ Lipids from cell membrane of hair fibers can be degraded by the visible light, but also by UV-A and by UV-B light, favoring the oxidation of hair. ${ }^{7}$ In consequence, it is important to found products that can replace the lipids that were lost in hair due to extrinsic or intrinsic factors.

On the basis of a previous work ${ }^{8}$ the biosurfactant evaluated in this work is composed by $64.2 \%$ fatty acids and $21.9 \%$ of proteins, showing a fatty acid composition similar to those found in human hair. In this way, the use of this natural surfactant, which is composed by $50-55.2 \%$ linolelaidic acid, 15.7-22.2\% oleic and/or elaidic acid, 5.9-14.6\% stearic acid, and $14.9-19.6 \%$ palmitic acid, could represent an interesting and ecofriendly alternative for cosmetic companies, to other surfactants derived from petrochemicals, used in shampoos and conditioners formulations. However, before incorporating this biosurfactant in hair formulations, it is required to evaluate its interaction with the hair and know if it can be adsorbed on that. The adsorption of this biosurfactant onto the hair will depend on its ionic charge and hence on its electrostatic interaction with the hair surface. Chemical surfactants are classified according to hydrophilic polar group as anionic, cationic, amphoteric (zwitterionic) and nonionic, although biosurfactants are usually classified according their polymeric structure as glycolipids; glycopeptides or lipopeptides. ${ }^{9}$ Normally, hair surface has a negative charge, so it adsorbs cationic surfactants. For this reason, hair conditioners and also hair 
sunscreens contain cationic surfactants, mainly quaternary ammonium salts, which absorb UV light, protecting hair surface from dryness and oxidation. Contrarily, shampoo formulations are composed by anionic surface-active agents, which can induce, in many cases, hair protein loss, hair dryness, opacity and difficulty of handling. ${ }^{\mathbf{1 0 , 1 1}}$ However, when hair is damaged, no chemical or physical treatment can recover its original aspect, so cosmetic hair formulations should be focused on preventing instead of repairing damaged hair.

Some authors ${ }^{3,12}$ have reported that the adsorption of cationic surfactants on hair can be affected by some factors, such as adsorption time, the presence of co-solutes, the molecular structure of surfactants, temperature and acidity of the liquid phase. Moreover, electrostatic interactions play an important role on the adsorption of substances in solid matrix with opposite charge. ${ }^{13}$ Ran et al. ${ }^{14}$ have suggested that the adsorption of quaternary ammonium surfactants on hair, take place through electrostatic interaction between the negative charges of the hair surface and the ammonium ions, increasing slowly with the higher equilibrium concentration of surfactant, although due to the relatively low charge density of hair surface, the adsorption amount in this region is relatively low.

In this study human hair was treated with an aqueous solution containing a biosurfactant, extracted from corn steep liquor (CSL), under different conditions of $\mathrm{pH}$, temperature and treatment time. Under the critical micellar concentration (CMC), the increase in the surface tension of a surfactant solution is directly proportional to the amount of biosurfactant adsorbed by hair. Thus, the dependent variable chosen for being evaluated was the hair capacity. Additionally, microscopic images of hair treated with biosurfactant solution or treated with water were provided in this work in order to elucidate if the biosurfactant, extracted from CSL, had some harmful effect on the external cuticle of hair.

\section{Materials and methods}

\section{Extraction of biosurfactant from corn steep liquor}

Corn steep liquor (CSL) provided by Santa Cruz Biotechnology, with a solid content of $50 \%$, was diluted in water up to $50 \mathrm{~g} \mathrm{~L}^{-1}$, and extracted with chloroform (ratio CSL solution: chloroform $1: 2$ ) at $56{ }^{\circ} \mathrm{C}$ during $60 \mathrm{~min}$. After that chloroform was evaporated by vacuum distillation and the biosurfactant was dissolved in water up to reach the its critical micellar concentration (CMC), following the protocol established by Vecino et al. ${ }^{15}$

Previous to dissolve the biosurfactant in water the density of the biosurfactant was established by measuring the proportion of mass $(\mathrm{m})$ to volume in $1 \mathrm{~mL}$ of sample. In addition, $\mathrm{pH}$ of biosurfactant extract was measured by using a $\mathrm{pH}$ meter Basic 20 (Crison, Spain).

\section{Determination of the critical micellar concentration of biosurfactant}

Under the CMC it can be established a linear relationship between the increase in the surface tension and the concentration of a specific biosurfactant in an aqueous solution. ${ }^{\mathbf{1 6 - 1 8}}$
Thus, for establishing this relationship, different solutions containing different concentrations of this biosurfactant were prepared and their surface tension was measured using a tensiometer Kruss EasyDyne, choosing the Wilhelmy plate method. Measurements were made in triplicate samples to increase the accuracy of the results.

\section{Fatty acid characterization of biosurfactant extract by GC-MS}

The fatty acid composition of the biosurfactant extracted from CSL was determined by gas chromatography (Trace GC Ultra, Thermo Scientific) coupled to a mass spectrometer (Trace DSQ, Thermo Finnigan), after methylation and trans-esterification of fatty acids into fatty acid methyl esters (FAMEs), according to the method described in the European Standard, ISO 129662:2011. FAMEs separation was performed on a ZB-WAX column $(60 \mathrm{~m} \times 0.25 \mathrm{~mm}$ i.d. $\times 0.25 \mu \mathrm{m}$ film thickness $)$ with an oven temperature gradient of $60^{\circ} \mathrm{C}$ for $2 \mathrm{~min}$, then $60-200^{\circ} \mathrm{C}$ at $10^{\circ} \mathrm{C}$ $\mathrm{min}^{-1}$, held for $27 \mathrm{~min}$, then increased to $240{ }^{\circ} \mathrm{C}$ at $5{ }^{\circ} \mathrm{C} \mathrm{min}^{-1}$, and finally held for $20 \mathrm{~min}$. Helium was used as carrier gas at a flow rate of $1 \mathrm{~mL} \mathrm{~min}^{-1}$, and the temperature of both injector inlet and the transfer line of detector was set at $250{ }^{\circ} \mathrm{C}$.

The mass spectra were obtained using a mass selective detector under electron impact ionization at a voltage of $70 \mathrm{eV}$, and data were acquired over an $\mathrm{m} / \mathrm{z}$ range of 40-400.

FAMEs were identified from a mass spectra library supplied with the GC-MS system and by comparison of retention times and mass spectra of a FAME standard mix, injected under the same conditions.

\section{Determination of the ionic nature of the biosurfactant using polymeric resins}

A solution containing $205.55 \mathrm{mg} \mathrm{L}^{-1}$ of biosurfactant was treated with STRATA XL-C, a cationic resin and STRATA X-A, an anionic resin. Previously to use STRATA XL-C was washed with 1 $\mathrm{mL}$ of methanol followed by $1 \mathrm{~mL}$ of water at $\mathrm{pH} 3.35$; whereas STRATA X-A was washed with $1 \mathrm{~mL}$ methanol followed by $1 \mathrm{~mL}$ of water at neutral $\mathrm{pH}$. After that $5 \mathrm{~mL}$ of biosurfactant solutions, at the CMC, were pass through the resins at $\mathrm{pH} 2.72$ and pH 6.74 for STRATA XL-C and STRATA X-A respectively.

\section{Hair surface preparation}

Samples of virgin hair from a 44 year male were used. The color of hair was dark brown and before using it, this was washed and rinsed out, until the surface tension of the water employed during the cleaning process achieved a value of $72 \mathrm{mN} \mathrm{m}^{-1}$. After that, the hair was dried for 24 hours, and kept in a paper envelope for its conservation. Its humidity was determined with the moisture analyzer Denver IR-60, being $12.94 \pm 0.33 \%$. Furthermore, its length was measured in order to use the more uniform sample as possible, so finally, samples of hair about 25 $\mathrm{mm}$ were used during the experiments.

\section{Adsorption experiments}

A Box-Behnken factorial design was established in order to study the interaction and adsorption of the lipopeptide 
biosurfactant extracted from corn onto human hair. The adsorption experiments were carried out using a hair surface/ biosurfactant relationship used of $1: 50(\mathrm{w} / \mathrm{v})$. Experiments were carried out in an orbital shaker at $200 \mathrm{rpm}$ using $100 \mathrm{~mL}$ flasks containing $5 \mathrm{~mL}$ of biosurfactant solution. At the time established by the experimental design, samples of biosurfactant solution were obtained and its surface tension was measured using the tensiometer described above. Following the surface tension of water was related with the concentration of biosurfactant, by using the calibration curve described previously, for the determination of biosurfactant CMC. The standardized (coded) dimensionless independent variables used, with limits of variation $(-1,1)$, were defined as $X_{1}$ (temperature), $X_{2}(\mathrm{pH})$ and $X_{3}$ (adsorption time); whereas the dependent variable $Y_{1}$ consisted of capacity of the hair to entrap biosurfactant depending on the experimental conditions. Regarding the variation range of the independent variables, temperature varied between $20^{\circ} \mathrm{C}$ and $50{ }^{\circ} \mathrm{C}$; $\mathrm{pH}$ between 5 and 7 and adsorption time between 2 and $30 \mathrm{~min}$.

\section{Statistical analysis}

The experimental data were analyzed by the response surface method with Statistica 7.0 software, by adjusting the dependent experimental data obtained to a quadratic function (eqn (1)); where $Y$ is the adsorption capacity of hair after the treatment, $\beta_{n}$ denotes the regression coefficients (calculated from experimental data by multiple regressions using the least-squares method) and $X$ denotes the independent variables (temperature, $\mathrm{pH}$, treatment time).

$$
\begin{aligned}
Y= & \beta_{0}+\beta_{1} X_{1}+\beta_{2} X_{2}+\beta_{3} X_{3}+\beta_{12} X_{1} X_{2} \\
& +\beta_{13} X_{1} X_{3}+\beta_{23} X_{2} X_{3}+\beta_{11} X_{1}^{2}+\beta_{22} X_{2}^{2}+\beta_{33} X_{3}^{2}
\end{aligned}
$$

In order to avoid that the independent variables selected in this study were influenced by their magnitudes, they were normalized and a relationship between coded and un-coded variables was established using linear equations, that were deduced from their respective variation limits (eqn (2)).

$$
X_{i}=\left(\frac{z_{i}-z_{i}^{0}}{\Delta z_{i}}\right) \beta_{\mathrm{d}}
$$

where $\Delta z_{i}$ is the distance between the real value in the central point and the real value in the superior or inferior level of a variable; $\beta_{\mathrm{d}}$ is the major coded limit value in the matrix for each variable, and $z_{i}^{0}$ is the real value in the central point. Coded variables were then assigned values of $-1,0$ and +1 , corresponding to the lowest, central and maximum limits of variation for each one.

\section{Microscopy hair analysis}

A microscopic study was performed with human hair samples to evaluate the possible changes in the surface morphology of hair fibers due to the adsorption of the biosurfactant. The microscopic used consisted of Olympus BX51; equipped with a highsensitivity camera Olympus DP71, a light source (U-RFL-T) Olympus, and photographs were taken using CellSens software.

\section{Results and discussion}

Samples of hair were treated with an aqueous solution containing $200 \mathrm{mg} \mathrm{L}^{-1}$ of biosurfactant, extracted from CSL. Fig. 1 shows an image of the biosurfactant concentrated, and then dissolved in water. The appearance of the biosurfactant after extraction is a yellow liquid with a density of $1.053 \mathrm{mg} \mathrm{mL}^{-1}$ and $\mathrm{pH}$ about 4. This biosurfactant is produced spontaneously in CSL in a process where the production of biosurfactant is not controlled. Hence, it is important to corroborate the composition of this biosurfactant each time that new corn steep liquor is used as a source of biosurfactant in order to verify its physicochemical properties before using it.

The biosurfactant extract used in this work was composed mainly by C18-C16 fatty acids, being the major fatty acid the linolelaidic acid followed by oleic and/or elaidic acid, with a similar composition than that established in previous works. ${ }^{\mathbf{8}}$ At concentrations higher the CMC the biosurfactant was able to reduce the surface tension of water from 72 up to $39 \mathrm{mN} \mathrm{m}^{-1}$ (see Fig. 2).

The advantage of this biosurfactant in comparison with the petrochemical surfactants, used in hair formulations, is based on its biocompatibility. Researches agree that many synthetic surfactants can damage the human scalp hair, producing irritation and allergy problems. ${ }^{\mathbf{1 , 1 9 2 0}}$ For example, lauryl sulfates are excellent cleansers, but hard on the hair, requiring careful selection of a secondary detergent and possible use of

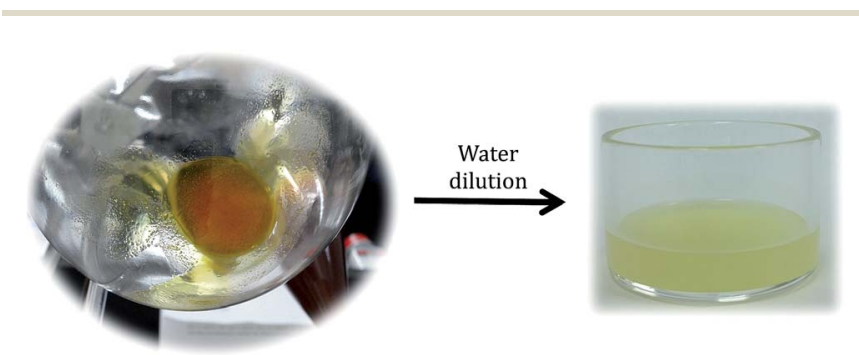

Fig. 1 Images of the biosurfactant extracted from CSL concentrated and diluted in water.

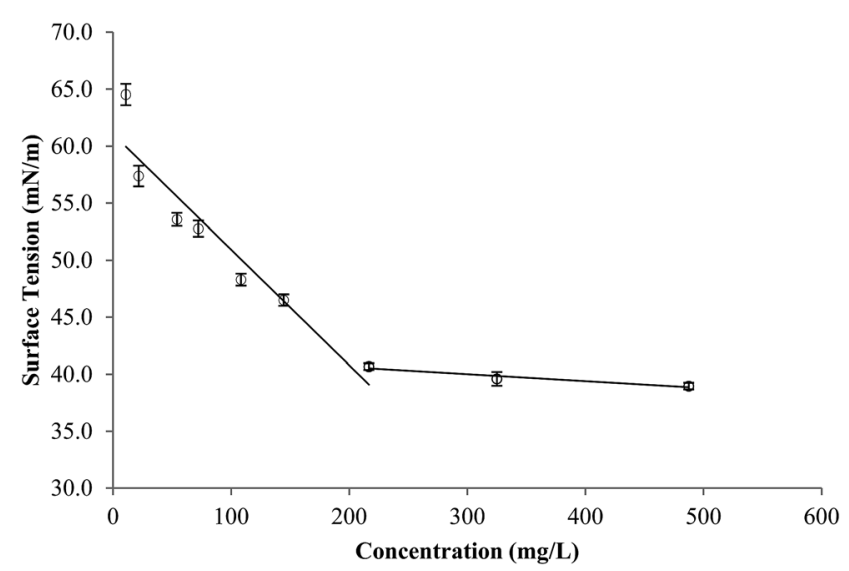

Fig. 2 Relationship between the surface tension of water and biosurfactant concentration. 
a conditioning agent as part of the shampoo formulation. ${ }^{21}$ Therefore, cosmetic industry demands more natural products that are compatible with the complex structure of hair fibers. The biosurfactant evaluate in this work provides an extract benefit as long as it can be adsorbed by the hair cell membranes because the cuticles, covering cortex inner, are composed of mostly fatty acid in their flat region and partly protein in their edges. ${ }^{3}$

Previously to the experiments with hair, the biosurfactant extracted from CSL, was treated with two kinds of resins (anionic and cationic), hence its ionic charge can be elucidated. It was observed that both resins entrap this biosurfactant. Therefore, it can be speculated that it is amphoteric, so it could be adsorbed by hair surface. The surface tension of biosurfactant solution after treatment with STRATA XL-C and STRATA X-A observing an increase of the surface tension since $41.3 \pm 0.2 \mathrm{mN} \mathrm{m}^{-1}$ to $69.3 \pm 5.5$ and $70.4 \pm 4.9 \mathrm{mN} \mathrm{m}^{-1}$, respectively.

Adsorption experiments using the cationic resins were carried out at $\mathrm{pH} 2.72$; whereas the treatment with the anionic resin was carried out at $\mathrm{pH}$ 6.74. For some amphoteric surfactants, the charge of the hydrophilic part is controlled by the $\mathrm{pH}$ of the solution, hence it can act as an anionic surfactant in an alkali solution or as a cationic surfactant in an acidic solution.

It is expected that the positively charged of the lipopeptide biosurfactant diffuse to the negative surface of hair. Thus it could be useful in the treatment of damaged hair, characterized by a higher number of negatively charged acid groups favoring the adsorption of positively charged surfactants. Moreover, with the adsorption of this biosurfactant on human hair, part of the lost fatty acids could be replaced obtaining a denser hair.

Therefore, after corroborating that the biosurfactant extracted from CSL is in part charged positively, it was included in an experimental design, in order to study its adsorption onto the hair under different conditions of $\mathrm{pH}$, temperature and extraction time. These independent variables were selected taking into account possible hair treatments. Hence during experiments $\mathrm{pH}$ varied between 5 and 7 , temperature between $20{ }^{\circ} \mathrm{C}$ and $50{ }^{\circ} \mathrm{C}$ and treatment time varied between 2 and 30 $\min$.

Table 1 shows the capacity of hair to adsorb the biosurfactant after treatment, under the different operational conditions established in the assay; whereas Table 2 shows the coefficients obtained after the statistical treatment of data.

Based on these coefficients, a relationship between the hair's capacity to entrap the biosurfactant and the independent variables selected during the treatment, in the range tested, can be established (see eqn (3)). This equation only includes those coefficients whose variables gave a significant effect on the adsorption of biosurfactant by hair, with a $p$ value $<0.05$. Table 1 also includes the theoretical capacity of hair, for adsorbing the lipopeptide biosurfactant established by eqn (3), under the conditions selected in the experimental design named $Y_{1}$. Additionally, for comparative purposes in Table 1 also were included the theoretical values, $Y_{1}(\mathrm{~A})$, achieved when all the coefficients (significant and no significant), provided in Table 2 were used to calculate the theoretical capacity; as well the
Table 1 Operational conditions considered in this study (expressed in terms of the coded independent variables: dimensionless temperature $X_{1}$, dimensionless $\mathrm{pH} X_{2}$ and dimensionless time $X_{3}$ ) and experimental results achieved for the dependent variable $Y_{1} . Y_{1}(\mathrm{~A})$ and $Y_{1}(\mathrm{~B})$ are the theoretical values obtained using the equation provided by the model taking into account all the coefficients, $Y_{1}(A)$, or just taking into account only the significant coefficients, $Y_{1}$ (B)

\begin{tabular}{|c|c|c|c|c|c|c|}
\hline \multirow[b]{2}{*}{ Exp. } & \multicolumn{3}{|c|}{$\begin{array}{l}\text { Independent } \\
\text { variable }\end{array}$} & \multicolumn{3}{|c|}{ Dependent variable } \\
\hline & $X_{1}$ & $X_{2}$ & $X_{3}$ & $Y_{1}$ & $Y_{1}(\mathrm{~A})$ & $Y_{1}(\mathrm{~B})$ \\
\hline 1 & 0 & -1 & -1 & 922.17 & 1138.33 & 703.113 \\
\hline 2 & 0 & 1 & -1 & 1118.74 & 1463.35 & 1292.07 \\
\hline 3 & 0 & -1 & 1 & 1680.70 & 1336.10 & 1164.82 \\
\hline 4 & 0 & 1 & 1 & 2405.14 & 2188.98 & 1753.78 \\
\hline 5 & -1 & -1 & 0 & 1115.53 & 2765.94 & 2598.12 \\
\hline 6 & -1 & 1 & 0 & 2159.36 & 2571.32 & 2403.50 \\
\hline 7 & 1 & -1 & 0 & 1143.49 & 731.53 & 563.71 \\
\hline 8 & 1 & 1 & 0 & 2644.46 & 2104.05 & 1936.23 \\
\hline 9 & -1 & 0 & -1 & 2546.16 & 1789.60 & 1654.17 \\
\hline 10 & -1 & 0 & 1 & 3678.67 & 3482.87 & 3347.44 \\
\hline 11 & 1 & 0 & -1 & 1574.53 & 1779.33 & 1634.91 \\
\hline 12 & 1 & 0 & 1 & 243.90 & 1000.46 & 865.04 \\
\hline 13 & 0 & 0 & 0 & 1371.68 & 1228.44 & 1228.44 \\
\hline 14 & 0 & 0 & 0 & 1148.91 & 1228.44 & 1228.44 \\
\hline 15 & 0 & 0 & 0 & 1164.73 & 1228.44 & 1228.44 \\
\hline
\end{tabular}

Table 2 Regression coefficients and their statistical significance for variable $Y_{1}$

\begin{tabular}{lrl}
\hline Coefficient & $Y_{1}$ & $p_{Y_{1}}$ \\
\hline$\beta_{0}$ & 1228.44 & $0.00340^{a}$ \\
$\beta_{1}$ & -625.42 & $0.00490^{a}$ \\
$\beta_{11}$ & 646.95 & $0.00985^{a}$ \\
$\beta_{2}$ & 294.48 & $0.02156^{a}$ \\
$\beta_{22}$ & 167.82 & 0.12198 \\
$\beta_{3}$ & 230.85 & $0.03438^{a}$ \\
$\beta_{33}$ & 135.43 & 0.17135 \\
$\beta_{12}$ & 391.79 & $0.02425^{a}$ \\
$\beta_{13}$ & -615.79 & $0.01003^{a}$ \\
$\beta_{23}$ & 131.97 & 0.16771 \\
${ }^{a}$ Significant coefficients $(p<0.05)$. & \\
\hline
\end{tabular}

theoretical values, $Y_{1}$ (B), calculated using only the significant coefficients of Table 2 . In both cases the differences between the experimental and theoretical results were similar, for most of the experimental conditions assayed.

$$
\begin{aligned}
Y_{1} & =1228.44-625.42 X_{1}+294.48 X_{2}+230.85 X_{3} \\
& +391.79 X_{1} X_{2}-615.79 X_{1} X_{3}+646.95 X_{1}^{2}
\end{aligned}
$$

Additionally, Fig. 3 shows a Vilfredo Pareto chart of standardized effects for $Y_{1}$, observing that temperature was the most influential variable on hair capacity, followed by the $\mathrm{pH}$; whereas the treatment time was the less influential variable. However, when temperature and extraction time are taking into account together, it was observed a significant effect on the adsorption capacity of biosurfactant by hair. 


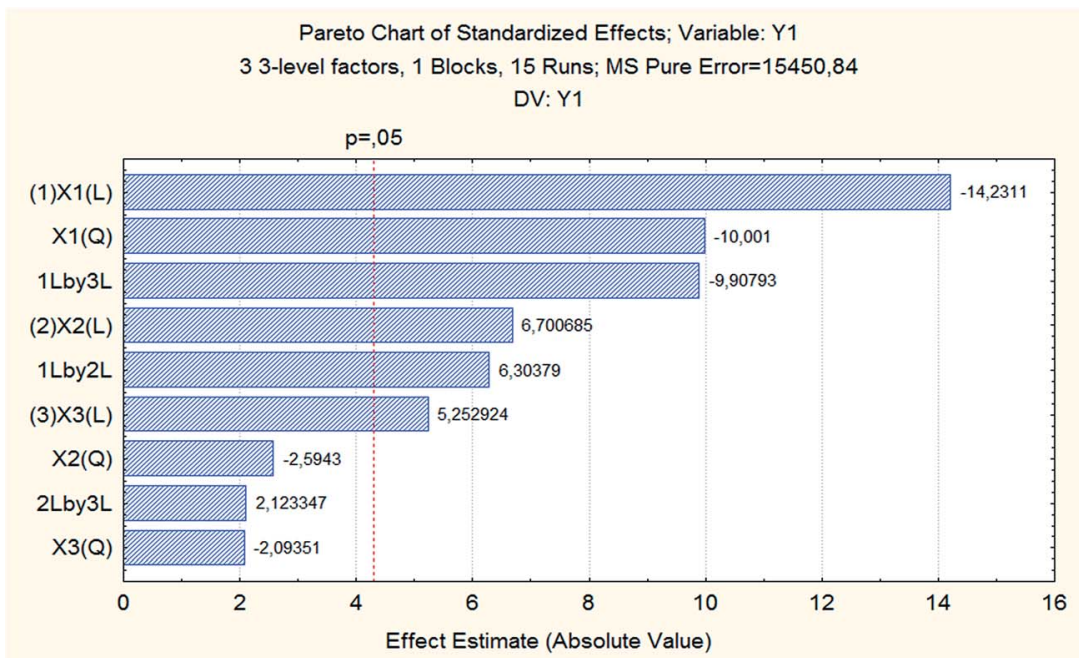

Fig. 3 Vilfredo Pareto chart of normalized effects for the establishment of the more influential independent variables on the adsorption capacity of hair.

Moreover, it was noticed that the interaction between temperature and $\mathrm{pH}$ also had a significant effect on the dependent variable studied. Additionally, Fig. 4 shows the correspondence between the experimental and theoretical results predicted by the model with $r^{2}$ value of 0.763 .

On the other hand, Fig. 5 shows the variation on hair capacity with the temperature and $\mathrm{pH}$, the most influential variables, fixing the treatment time at the lowest (Fig. 5A), and highest (Fig. 5B) values respectively, established in the experimental design.

When the treatment of hair was carried out during $2 \mathrm{~min}$, the model predicts that hair can adsorb between 1200 and $2363 \mu \mathrm{g}$ of biosurfactant per $\mathrm{g}$ of hair, observing that higher temperatures and higher $\mathrm{pH}$, in the range tested, increase the adsorption capacity of hair; although at low temperature the $\mathrm{pH}$ had a lower significant effect on the capacity (Fig. 5A). However, when the treatment time was increased up to $16 \mathrm{~min}$ it was observed that lower temperatures had a positive effect on the hair adsorption capacity, noticing that $\mathrm{pH}$ only had negative influence in the adsorption capacity between middle and higher temperatures. Hence, when hair treatment was carry out at intermediate time (16 min), the model predicts that the capacity of hair is between 892 and $2578 \mu \mathrm{g} \mathrm{g}^{-1}$; whereas when the treatment time was fixed at $30 \mathrm{~min}$, also maximum capacities were obtained at low temperature. In this case, pH only presented a significant effect on hair capacity when temperature was increased. After $30 \mathrm{~min}$ of treatment the model predicts capacities between 653 and $3382 \mu \mathrm{g} \mathrm{g}^{-1}$ (Fig. 5B).

The capacities values obtained for the adsorption of the biosurfactant on hair is in the range than that obtained by Ran et al. ${ }^{14}$ in hair assays, using a cationic surfactant consisting of quaternary ammonium surfactant (DDABDT). These authors studied the adsorption of this derived petrochemical

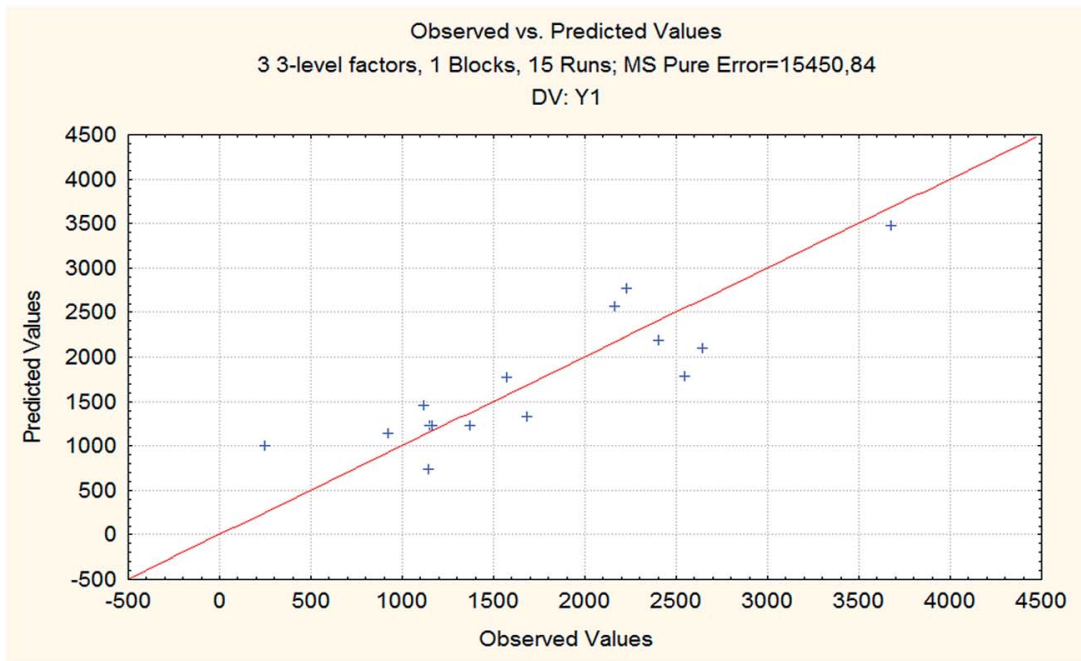

Fig. 4 Correspondence between experimental and theoretical values for variable $Y_{1}$. 


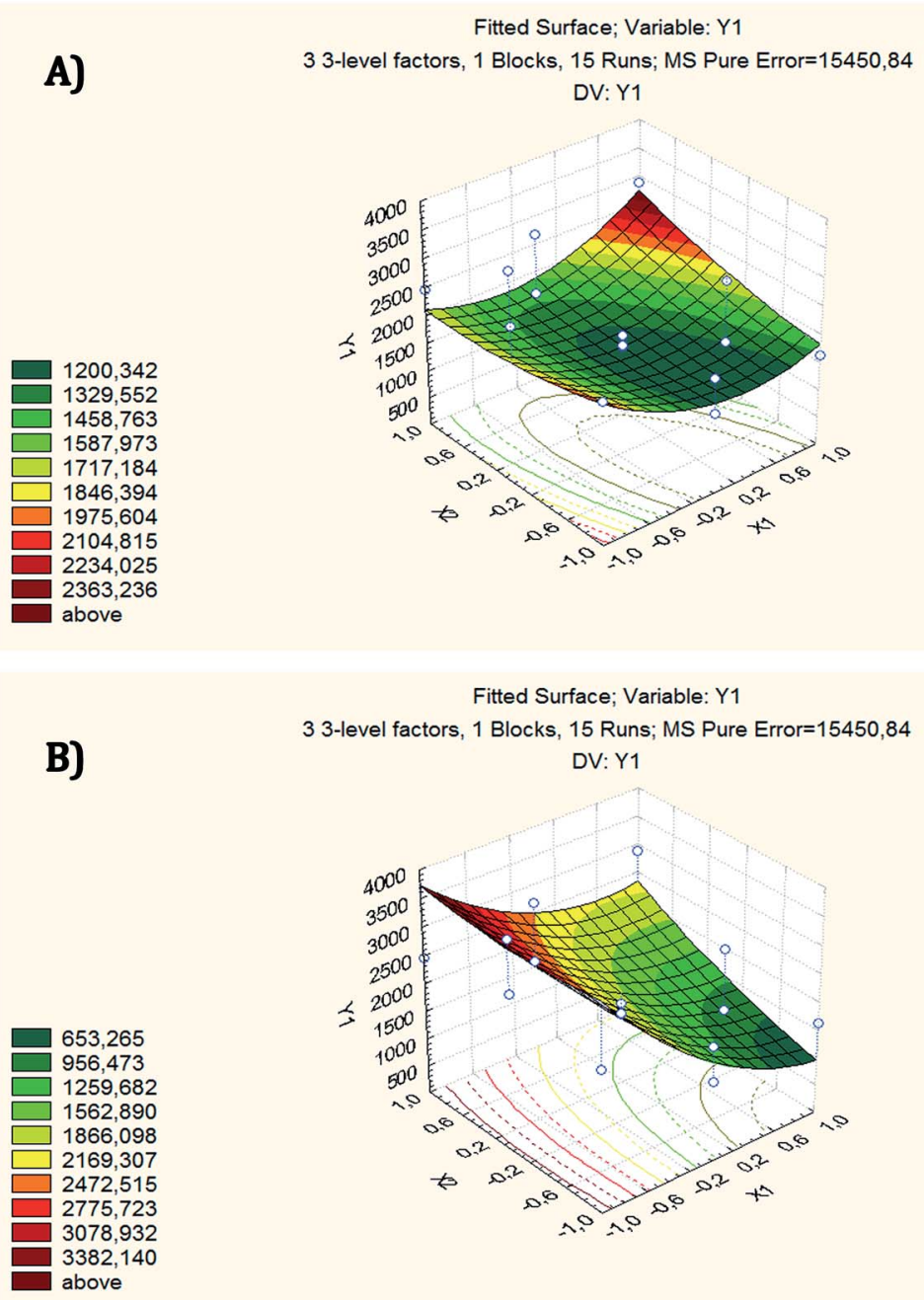

Fig. 5 Variation in the adsorption capacity of hair depending on variables $X_{1}$ (temperature) and $X_{2}(\mathrm{pH})$, fixing $X_{3}$ (time), at the lowest value (A) and highest value (B).

surfactant on hair under different temperatures and different concentrations of surfactant. They used concentrations of DDABDT between 24.5 and $236 \mathrm{mg} \mathrm{L}^{-1}$, corresponding with treatments carried out using $8 \%$ of hair. At the lowest concentration of surfactant assayed they obtained capacities about $1500 \mu \mathrm{g} \mathrm{g}^{-1}$, after $60 \mathrm{~min}$ of treatment; whereas when the concentration of surfactant was fixed about $236 \mathrm{mg} \mathrm{L}^{-1}$ they obtained capacities around $4500 \mu \mathrm{g} \mathrm{g}^{-1}$. In the current assay, the concentration of biosurfactant was established below the CMC (200 $\left.\mathrm{mg} \mathrm{L}^{-1}\right)$ and the amount of hair was fixed in $2 \%$ $(\mathrm{w} / \mathrm{w})$. The maximum experimental capacity obtained was 3679 $\mu \mathrm{g} \mathrm{\textrm {g } ^ { - 1 }}$, that it was close to the maximum theoretical capacity predicted by the model $\left(3382 \mu \mathrm{g} \mathrm{g}^{-1}\right)$. These values are in the range of the values obtained by Ran et al. ${ }^{14}$ for the cationic surfactant DDABDT.

When Ran et al. ${ }^{14}$ studied the adsorption of DDABDT on cuticles of scalp hair, they observed that at the beginning of the process, the adsorption of DDABDT was very fast. These results are in concordance with the fast diffusion observed for the biosurfactant evaluated in this work on the surfaces of hair fibers, observing that treatment time had a lower significant effect on the adsorption of biosurfactant onto hair. Additionally, these authors found that the concentration of surfactant is an important aspect during the treatment of hair. They found that at concentrations of surfactant higher than the CMC the capacity of hair to entrap the surfactant increased, in comparison with adsorption experiments carried out with concentrations of surfactant lower the CMC. They reported that the association between hair and surfactant could be structured as monolayer or bilayer, depending on the initial concentration of the surfactant. In the monolayer structure, Ran et al., ${ }^{14}$ suggest that surfactant molecules are adsorbed vertically on hair surface fibers through electrostatic interaction; whereas in the bilayer structure, the second layer molecules may then pile up on top of the first layer with charged groups orienting outward. The biosurfactant solution used in this work was used under the CMC, thus the association between the biosurfactant and hair should be monolayer. This fact can explain that only part of the lipopeptide biosurfactant is adsorbed by hair. Ran et al. ${ }^{14}$ obtained percentages of 

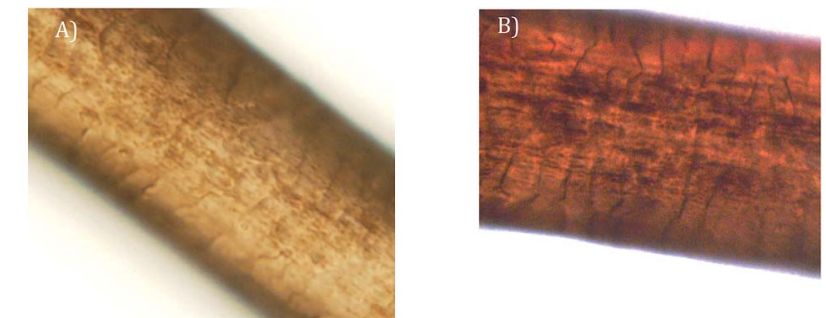

Magnification 60x

Fig. 6 Microscope images of human hair surface without biosurfactant (A) and with a higher adsorption of biosurfactant (B).

surfactant adsorption onto hair between 12 and 36\%, similar to the adsorption percentages obtained in the current work with the biosurfactant extracted from CSL (10-44\%).

Fig. 6 shows the microscope images of hair surface corresponding to different treatments applied in the current work. Fig. 6A shows images of hair washed with water (control) whereas Fig. 6B shows sample of hair characterized by a higher adsorption of biosurfactant (experiment 10). Small differences were visualized between samples, observing that the treatment with the biosurfactant almost did not affect to the surface morphology of hair; just it was noticed that those samples of hair that adsorbed more biosurfactant, had a more defined cuticle. No residues of biosurfactant were detected in the hair surface.

\section{Conclusions}

Based on the results obtained in this work the biosurfactant extracted from CSL could represent an interesting and ecofriendly alternative to cationic and amphoteric surfactants derived by petrochemicals, that although make hair easier to comb and manage, and have anti-static properties they are potentially strong irritants, and are hence only used with less irritating nonionic surfactants in shampoos designed for chemically-treated or very dry hair.

Further experiments will be needed, using concentrations of biosurfactant, above the CMC, in order to know if the formation of biosurfactant molecule aggregates can increase the adsorption of biosurfactant onto the hair surface, although if it probably occurs this association, should be weaker than if the adsorption of biosurfactant on hair is in monolayers. In addition, it would be interesting to study the differences obtained during the adsorption process of this biosurfactant using damaged and undamaged human hair.

\section{Acknowledgements}

This study was supported by the Spanish Ministry of Economy and Competitiveness under the project CTM2015-68904 (FEDER funds). L. Rodríguez-López acknowledges to the University of Vigo (Spain) for her pre-doctoral fellowship, and X. Vecino is grateful for her post-doctoral grant (SFRH/BPD/ 101476/2014) financed by the Portuguese Foundation for Science and Technology (FCT, Portugal).

\section{References}

1 R. M. Trüeb, Z. Hautkrankh., 2007, 6, 356.

2 J. Clarke, C. Robbins and B. Schroff, J. Soc. Cosmet. Chem., 1989, 40, 309.

3 R. Guoxia, Y. Zhang, Q. Song, Y. Wang and D. Cao, Colloids Surf., B, 2009, 68, 106.

4 C. R. Robbins, Chemical and Physical Behaviour of Human Hair, Springer, Berlin, 2012.

5 R. I. Logan, D. E. Rivett, J. D. Tucker and A. H. F. Hudson, Text. Res. J., 1989, 59, 109.

6 J. S. Capablanca and I. C. Watt, Text. Res. J., 1986, 56, 49.

7 E. Hoting and M. Zimmermann, J. Cosmet. Sci., 1997, 48, 79.

8 X. Vecino, L. Barbosa-Pereira, R. Devesa-Rey, J. M. Cruz and A. B. Moldes, J. Agric. Food Chem., 2014, 62, 5451.

9 R. Marchant and I. M. Banat, Biotechnol. Lett., 2012, 34, 1597. 10 R. D. C. C. Wagner and I. Joekes, Colloids Surf., B, 2005, 41, 7. 11 C. Barba, S. Scott, R. Kelly, J. L. Parra and L. Coderch, J. Appl. Polym. Sci., 2010, 115, 1461.

12 M. J. Rosen, Surfactants and Interfacial Phenomena, John Wiley and Sons, New York, 1978, vol. 16.

13 S. Paria and K. C. Khilar, Adv. Colloid Interface Sci., 2004, 110, 75.

14 G. Ran, Y. Zhang, Q. Song, Y. Wang and D. Cao, Colloids Surf., B, 2009, 68, 106.

15 X. Vecino, L. Barbosa-Pereira, R. Devesa-Rey, J. M. Cruz and A. B. Moldes, Bioprocess Biosyst. Eng., 2015, 38, 1629.

16 S. H. Kim, E. J. Lim, S. O. Lee, J. D. Lee and T. H. Lee, Biotechnol. Appl. Biochem., 2000, 31, 249.

17 L. R. Rodrigues, J. A. Teixeira, H. C. Van der Mei and R. Oliveira, Colloids Surf., B, 2006, 53, 105.

18 S. De, S. Malik, A. Ghosh, R. Saha and B. Saha, RSC Adv., 2015, 5, 65757.

19 W. F. Bergfeld, The side effects of hair products on the scalp and hair, Springer, Berlin Heidelberg, 1981.

20 R. M. Trüeb, J. Invest. Dermatol. Symp. Proc., 2005, 10, 289.

21 Z. D. Draelos, Int. J. Trichology, 2010, 2, 24. 